\title{
Acoustic mode radiation from the termination of a truncated nonlinear internal gravity wave duct in a shallow ocean area
}

\author{
Ying-Tsong Lin, Timothy F. Duda, and James F. Lynch \\ Department of Applied Ocean Physics and Engineering, Woods Hole Oceanographic Institution, Woods \\ Hole, Massachusetts 02543
}

(Received 26 January 2009; revised 20 June 2009; accepted 8 July 2009)

\begin{abstract}
Horizontal ducting of sound between short-wavelength nonlinear internal gravity waves in coastal environments has been reported in many theoretical and experimental studies. Important consequences arising at the open end of an internal wave duct (the termination) are examined in this paper with three-dimensional normal mode theory and parabolic approximation modeling. For an acoustic source located in such a duct and sufficiently far from the termination, some of the propagating sound may exit the duct by penetrating the waves at high grazing angles, but a fair amount of the sound energy is still trapped in the duct and propagates toward the termination. Analysis here shows that the across-duct sound energy distribution at the termination is unique for each acoustic vertical mode, and as a result the sound radiating from the termination of the duct forms horizontal beams that are different for each mode. In addition to narrowband analysis, a broadband simulation is made for water depths of order $80 \mathrm{~m}$ and propagation distances of $24 \mathrm{~km}$. Situations occur with one or more modes absent in the radiated field and with mode multipath in the impulse response. These are both consistent with field observations.

(C) 2009 Acoustical Society of America. [DOI: 10.1121/1.3203268]
\end{abstract}

PACS number(s): 43.30.Re, 43.20.Bi, 43.20.Mv, 43.30.Bp [JAC] Pages: 1752-1765

\section{INTRODUCTION}

Theoretical and experimental studies have revealed that propagating sound can be trapped and ducted between pairs of nonlinear internal waves of depression commonly observed in coastal areas. Here, we examine what happens when such a duct gradually or abruptly relaxes to the background conditions and the sound exits the duct. The horizontal ducting of sound between internal waves can be understood in terms of the horizontal rays of acoustic vertical modes bending and focusing in the duct, or by spiraling rays. Katsnel'son and Pereselkov ${ }^{1}$ theoretically studied the effect using the theory of "horizontal rays and vertical modes." Another study by Oba and Finette ${ }^{3}$ which included results obtained with a computer code for three-dimensional (3D) sound propagation, FOR3D, ${ }^{4}$ also predicted this ducting phenomenon. In another of their papers, ${ }^{5}$ these investigators numerically investigated the consequences of this effect on horizontal array beamforming and also performed a modal decomposition of the sound field to explain its structure in the duct. Observations by Badiey et al. ${ }^{6}$ and Franks et al. ${ }^{7}$ from the SWARM'95 experiment off New Jersey confirmed these predictions. Typical internal wave packets capable of causing this effect have inter-wave distances of 300-1000 m and modal refractive index anomalies of a few tenths of a percent to $3 \%{ }^{8}$

Work to date has addressed these internal wave ducts as "infinite length pipelines" for sound, i.e., the internal wave crests are straight and endless. However, this is not the case in the real world. Many satellite and in-situ observations have shown internal wave packets (ducts) to be finite and localized, having total along-crest lengths as short as a few kilometers near an internal wave source region. ${ }^{9}$ Prior work does not address what happens to internal-wave ducted sound when the duct terminates in the horizontal and the sound is emitted. The goal of this paper is to investigate this radiation from the duct termination. Analytical and numerical approaches are taken. In the analytical approach, an idealized internal-wave shape (square waveform) and the assumption of adiabatic mode propagation give a 3D normal mode solution for the sound field in the duct. Then Huygens' principle is adopted to calculate the radiation field. As for the numerical approach, a computer code ${ }^{10}$ implementing a $3 \mathrm{D}$ parabolic approximation in Cartesian coordinates is employed to calculate the acoustic field and broadband impulses within and emitted from a duct. The use of this 3D parabolic approximation model allows more complicated (or more realistic) internal-wave shapes. Also, since this numerical model allows mode coupling, it can be used to verify the adiabatic mode assumption used in the analytical computation.

Both the analytical and numerical computations show horizontal interference patterns within the duct. Richly detailed sound radiation fields are predicted at locations far from the termination of a truncated internal wave duct, having mode-dependent patterns with strong azimuthal and temporal variability. Thus, effects of ducting are found at locations with no evidence of a ducting condition. Similar radiation effects were found in the data collected in the SW06 shallow water acoustic experiment. ${ }^{11}$ Though we will not completely prove it in this paper (as this involves other analyses which are future work), the results shown here make a plausible explanation for at least some of the data variability measured in the field. As examples, the tempo- 
(a) Internal square wave model

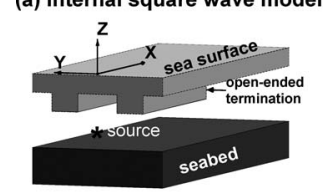

(b) Vertical mode comparisons

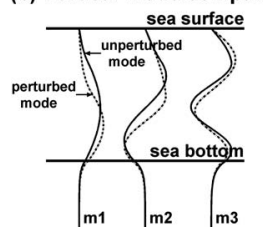

Radiation beam pattern of a vertical propagating mode (Total radiation field $=$ sum of all vertical mode radiations)

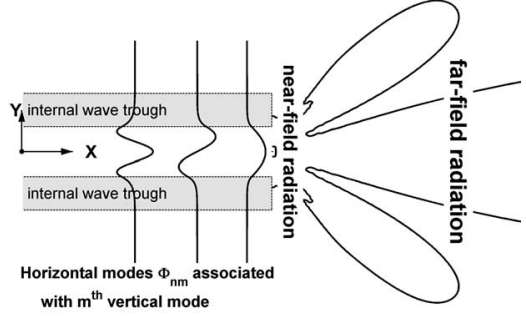

(c) Sound radiation from the termination

FIG. 1. Simplified internal-wave duct model used for theoretical analysis. (a) Internal waves of depression with square waveforms and parallel wavefronts are considered. The waves abruptly terminate to the background state. (b) Comparisons of acoustic vertical modes before and after being perturbed by the internal-square wave. (c) A depiction of sound radiation from the termination. Total radiation field equals to the sum of all vertical mode radiations.

rally varying modal contents of sound and mode multipath were observed and compare well qualitatively with the predictions made here.

The contents of this paper are as follows: Sec. II contains the derivation and evaluation of a 3D normal mode solution for an idealized environment. Section III contains a numerical parabolic equation solution for a more complex environment. Section IV contains field observation of the effects similar to those predicted by the theory. Section V has a discussion and Sec. VI contains a summary.

\section{NORMAL MODE SOLUTION}

The horizontal ducting of acoustic energy between internal waves can lead to a rather complicated horizontal eigenvalue problem if realistic detailed shapes of the internal waves that form the duct are considered. Also, the sound radiation from a real, irregular duct termination is not simple to handle by a theoretical means. So, rather than addressing a complicated case, we will begin with a simple duct model which will still retain most of the physics associated with the ducting and radiation effects. The detailed model configuration is provided below, along with the explanations for two assumptions used in deriving the normal mode solution.

\section{A. Simplified physical model and assumptions}

The model used for the theoretical analysis has two homogeneous water column layers bounded below by a homogeneous bottom, as shown in Fig. 1(a). The sound speed in the upper water column layer is slightly faster, and sound absorption is neglected in both the water column layers, but is included in the bottom. Two internal waves of depression are introduced by deepening the interface between the two aqueous layers from the ambient state to a perturbed state. Simplified internal waves with square and equal waveforms in the $y$ direction, equal perturbation amplitudes, and parallel wave crests along the $x$-axis are used, forming an acoustic duct with slow normal velocity in the center, as will be shown. The waves abruptly terminate at a selected location in the $x$ direction, forming an open end of the duct. Although

the acoustic horizontal ducting modes in this square-wave approximate model may differ from the ones seen in realistic cases, the physical characteristics of these ducting modes can still be captured in the approximate model. In addition, if it is required, one can more carefully design an internal squarewave model to fit a realistic waveform such that the resultant horizontal ducting modes are comparable to the realistic ones. The coordinate origin of the Cartesian coordinate system is placed in the middle between the internal waves on the sea surface. The $z$ axis is positive upward. At $x$ locations less than termination position $x=L$ solutions valid in a ducted environment will be found. At $x>L$ solutions in a homogeneous layered environment will be computed using Huygens' principle, which is done by employing the Green's function method.

For the theoretical analysis, we make two following assumptions about sound propagation. (1) Mode propagation is adiabatic, with no energy exchange between modes. This assumption would be invalid if the addressed problem was sound propagation across the internal waves. ${ }^{12,13}$ (2) Total transmission at the open-ended termination is assumed so that no sound energy is reflected back into the duct. This is justified by the fact that the termination width normal to the duct $(300-1000 \mathrm{~m}$ across in a common situation) is very large compared to the underwater acoustic wavelength of frequencies greater than $50 \mathrm{~Hz}$, which produces a large $k a$ value (acoustic wavenumber multiplying the gap aperture) and thus should yield only tiny reflected sound by analogy with the unflanged, open pipe problem. ${ }^{14}$ A final caution should be made here: for high grazing-angle penetration (at $x$ near that of the source) the assumption of adiabatic mode propagation is violated, and the solution in terms of the resultant mode sum is not strictly correct. The horizontal leaky modes are included in the following derivation, but they only describe the barrier penetration and tunneling effect that occurs when the trapped sound strikes the internal waves at low grazing angle and radiates out of the duct in the $y$ direction.

\section{B. Derivation of an analytical solution}

The sound pressure field excited by a harmonic point source located at $\left(x_{s}, y_{s}, z_{s}\right)$ in the physical model described above is governed by the inhomogeneous 3D Helmholtz equation with a Dirac delta source function

$$
\begin{aligned}
\rho(z) & \nabla \cdot\left(\frac{1}{\rho(z)} \nabla P(x, y, z)\right)+\frac{\omega^{2}}{c^{2}(x, y, z)} P(x, y, z) \\
& =-4 \pi \delta\left(x-x_{s}\right) \delta\left(y-y_{s}\right) \delta\left(z-z_{s}\right),
\end{aligned}
$$

where $c(x, y, z)$ is the medium sound speed (this is a complex number with imaginary component enumerating absorption), $\rho(z)$ is the medium density (its horizontal variations are neglected), and $\omega=2 \pi f$ is the acoustic frequency ( $f$ enumerates this in Hertz). To solve Eq. (1), begin with a vertical mode decomposition of the sound field

$$
P(x, y, z)=\sum_{m} \Gamma_{m}(x, y) \Psi_{m}(x, y, z),
$$

where $\Gamma_{m}(x, y)$ is the (complex) mode amplitude and $\Psi_{m}(x, y, z)$ is the $m$ th vertical normal mode at $(x, y)$ that 
satisfies the following local mode equation with appropriate boundary conditions on the sea surface and the bottom:

$$
\begin{aligned}
& \rho(z) \frac{d}{d z}\left(\frac{1}{\rho(z)} \frac{d}{d z} \Psi_{m}(x, y, z)\right)+\left(\frac{\omega^{2}}{c^{2}(x, y, z)}\right. \\
&\left.-\zeta_{m}^{2}(x, y)\right) \Psi_{m}(x, y, z)=0,
\end{aligned}
$$

where $\zeta_{m}$ is the horizontal wavenumber of the $m$ th vertical mode. Deriving analytic expressions for such vertical modes in the simplified, three-layer model considered here can be done by matching the interface conditions, and the details are omitted here. As shown in Fig. 1(b), the vertical mode functions are perturbed by the internal waves that comprise the duct. Also, the horizontal wavenumbers of vertical modes may be affected by the internal waves.

After replacing the sound pressure function in the 3D Helmholtz wave equation with the vertical mode decomposition and employing the assumption of adiabatic mode propagation, one can see that the vertical modal amplitude is governed by a two-dimensional (2D) Helmholtz equation, which is often called the horizontal refraction equation, ${ }^{15}$ given by

$$
\begin{aligned}
\left(\frac{\partial^{2}}{\partial x^{2}}+\frac{\partial^{2}}{\partial y^{2}}\right) \Gamma_{m}+\zeta_{m}^{2}(x, y) \Gamma_{m} \\
=-4 \pi \frac{\Psi_{m}\left(x_{s}, y_{s}, z_{s}\right)}{\rho\left(z_{s}\right)} \delta\left(x-x_{s}\right) \delta\left(y-y_{s}\right) .
\end{aligned}
$$

This equation was employed in Ref. 5 for studying the focusing/defocusing structure in a straight and endless internal wave duct. Note how the horizontal wavenumber of the $m$ th vertical mode, $\zeta_{m}$, mimics the wavenumber $k=\omega / c$ in Eq. (1). In addition, the internal waves may alter the values $\zeta_{m}$, yielding interface/boundary conditions across the waves in the $y$ direction. In the model considered here the internal waves of depression have perturbed wavenumbers that are lower than the unperturbed values so that an incident vertical mode from the inside of the duct impinging horizontally onto the internal waves may encounter a situation where total reflection occurs and vertical modes are ducted in the horizontal plane, as shown in Fig. 1(c).

Solving the horizontal refraction equations for the vertical modal amplitudes allows the sound pressure field to be computed via mode summation. A two-step procedure is implemented. The first step is to obtain the ducted sound field between the simplified internal waves, and the second step is to apply Huygens' principle to obtain the radiation field due to the sound pressure distributed at the open-ended termination.

\section{Ducted sound field}

We begin by solving for the field at $x \leq L$, where ducted horizontal modes will exist. To solve Eq. (4) in this region, we can utilize many of the methods used in solving boundary value problems in shallow water acoustics, such as the wellknown Pekeris waveguide mode problem. But, instead of looking at the constructive interferences of up- and downgoing plane waves to get vertical modes, we are now seeking horizontal modes associated with each vertical mode $\Psi_{m}$. Due to barrier penetration and tunneling effects, which cause sound transmission through the internal wave "walls" and radiation outward in the $y$ direction, Eq. (4) is not a proper Sturm-Liouville problem, so we cannot use an exact eigenfunction expansion to generate a solution. Instead, a wavenumber integration technique is employed, and a generalized eigenfunction expansion can be achieved for the vertical modal amplitude $\Gamma_{m}$ if the branch-line integral is neglected. The detailed derivation is described below. Beforehand, note that the generalized expansion is of the form

$$
\Gamma_{m}(x, y)=\sum_{n} A_{n m}(x) \Phi_{n m}(y),
$$

where $\Phi_{n m}$ are the horizontal modes associated with each vertical mode $\Psi_{m}$, and $A_{n m}$ are their (complex) amplitudes. A combination of indices $(n, m)$ is required for the horizontal ducted modes. The complete solution for the ducted sound field will be of the form $P(x, y, z)=\Sigma_{m} \Sigma_{n} A_{n m}(x) \Phi_{n m}(y)$ $\times \Psi_{m}(x, y, z)$. The horizontal mode functions only depend on $y$ since the environment in the duct area does not have $x$-dependency. The amplitude $A_{n m}$ depends on $x$ only and will mainly vary due to modal phase delays and modal attenuation, with no geometric spreading loss for the trapped modes.

The wavenumber integration technique of solving Eq. (4) for the vertical modal amplitude $\Gamma_{m}$ at $x \leq L$ is now described in detail. First, the assumption of total transmission at the open-ended termination allows us to reformat the problem to be an infinitely long duct problem, and the solution of the reformatted problem at the positions where $x \leq L$ (the internal waves terminate at $x=L$ ) is valid for representing the ducted sound field. Note that after we get the radiation field solution in Sec. II B 2, we will justify the assumption of total transmission. Since the horizontal wavenumber of the $m$ th vertical mode, $\zeta_{m}$, does not have $x$-dependency in the reformatted problem, we can apply the following plane-wave spectral integral or spatial Fourier transform to decompose the vertical modal amplitude in terms of a set of infinite plane waves:

$$
\begin{aligned}
& \Gamma_{m}(x, y)=\frac{1}{\sqrt{2 \pi}} \int_{-\infty}^{\infty} G_{m}\left(k_{x}, y\right) e^{i k_{x} x} d k_{x}, \\
& G_{m}\left(k_{x}, y\right)=\frac{1}{\sqrt{2 \pi}} \int_{-\infty}^{\infty} \Gamma_{m}(x, y) e^{-i k_{x} x} d x,
\end{aligned}
$$

where $G_{m}\left(k_{x}, y\right)$ is the plane-wave component of the $m$ th vertical modal amplitude with wavenumber $k_{x}$. Applying the inverse transform operator to both sides of Eq. (4) yields a one-dimensional, $y$-dependent Helmholtz equation that is comparable to the depth-dependent Green's function equation in the wavenumber integration technique, ${ }^{15}$

$$
\begin{aligned}
& \frac{d^{2}}{d y^{2}} G_{m}\left(k_{x}, y\right)+\left(\zeta_{m}^{2}-k_{x}^{2}\right) G_{m}\left(k_{x}, y\right) \\
& =-2 \sqrt{2 \pi} \frac{\Psi_{m}\left(x_{s}, y_{s}, z_{s}\right)}{\rho\left(z_{s}\right)} e^{-i k_{x} x_{s}} \delta\left(y-y_{s}\right),
\end{aligned}
$$

which is subject to interface conditions on the internal-wave 
"walls" that can be expressed as plane-wave reflection coefficients.

To determine $G_{m}\left(k_{x}, y\right)$ (and thence $\Gamma_{m}$ ) for $x \leq L$, we first obtain a solution for the domain bounded by the internal waves $-D / 2 \leq y \leq D / 2$, where $y= \pm D / 2$ are the inner boundaries of the internal waves, and then extend the bounded domain solution to the external domain by matching the interface condition at $y= \pm D / 2$. Utilizing the endpoint method, ${ }^{16}$ the plane-wave component $G_{m}\left(k_{x}, y\right)$ in the bounded domain $(|y| \leq D / 2)$ can be found as

$$
G_{m}\left(k_{x}, y\right)= \begin{cases}i \sqrt{2 \pi} \frac{\Psi_{m}\left(x_{s}, y_{s}, z_{s}\right)}{\rho\left(z_{s}\right)} e^{-i k_{x} x_{s}} \frac{\left[e^{-i k_{y} y}+R_{L m} e^{i k_{y} D} e^{i k_{y} y}\right]\left[R_{R m} e^{i k_{y} D} e^{-i k_{y} y_{s}}+e^{i k_{y} y_{s}}\right]}{k_{y}\left[1-R_{L m} R_{R m} e^{2 i k_{y} D}\right]} & \text { for }-D / 2 \leq y \leq y_{s}, \\ i \sqrt{2 \pi} \frac{\Psi_{m}\left(x_{s}, y_{s}, z_{s}\right)}{\rho\left(z_{s}\right)} e^{-i k_{x} x_{s}} \frac{\left[e^{-i k_{y} y_{s}}+R_{L m} e^{i k_{y} D} e^{i k_{y} y_{s}}\right]\left[R_{R m} e^{i k_{y} D} e^{-i k_{y} y}+e^{i k_{y} y}\right]}{k_{y}\left[1-R_{L m} R_{R m} e^{2 i k_{y} D}\right]} & \text { for } y_{s} \leq y \leq D / 2,\end{cases}
$$

where $k_{y}^{2}=\zeta_{m}^{2}-k_{x}^{2}$, and $R_{L m}$ and $R_{R m}$ are the plane-wave reflection coefficients at $y=-D / 2$ and $D / 2$, respectively, as a function of $k_{x}$ and the $m$ th vertical modal phase speeds inside and outside the internal waves. The plane-wave reflection coefficients can be obtained using the usual formulas for plane-wave reflections from horizontally stratified multilayered media. Note that there is no horizontal density contrast in the system we are considering. To complete the solution for the vertical modal amplitude $\Gamma_{m}$, we insert Eq. (8) into Eq. (6a) and employ a complex contour integration technique. This immediately gives us the equation for the eigenvalues:

$$
1-R_{L m} R_{R m} \exp \left(2 i \sqrt{\zeta_{m}^{2}-k_{x}^{2}} D\right)=0 .
$$

Since the plane-wave reflection coefficients $R_{L m}$ and $R_{R m}$ depend on which vertical mode is being considered, solving this eigenvalue equation leads to a set of horizontal mode wavenumbers for each vertical mode considered. There are two kinds of horizontal modes: trapped modes forming the ducted sound field and leaky modes forming the sound penetrating the internal waves and radiating laterally outward in the $y$ direction. By selecting the Pekeris branch cut and neglecting the branch-line integral, the vertical modal amplitude $\Gamma_{m}$ in the domain bounded by the internal waves $(-D / 2 \leq y \leq D / 2)$ is found to be

$$
\Gamma_{m}(x, y)=2 \pi i \frac{\Psi_{m}\left(x_{s}, y_{s}, z_{s}\right)}{\rho\left(z_{s}\right)} \sum_{n} \frac{\Phi_{n m}\left(y_{s}\right)}{\kappa_{n m} w_{n m}} e^{i \kappa_{n m}\left(x-x_{s}\right)} \Phi_{n m}(y),
$$

where $w_{n m}$ is the normalization factor for the unnormalized horizontal mode function $\Phi_{n m}$,

$$
\begin{aligned}
w_{n m}=i[ & \left.d\left(1-R_{L m} R_{R m} e^{2 i k_{y} D}\right) / d k_{y} \mid \begin{array}{c}
k_{y}^{2}=\zeta_{m}^{2}-k_{x}^{2} \\
k_{x}=\kappa_{n m}
\end{array}\right] \\
\times & {\left[R_{L m} e^{i \sqrt{\zeta_{m}^{2}-\kappa_{n m}^{2}} D}\right], } \\
\Phi_{n m}(y)= & {\left[e^{-i \sqrt{\zeta_{m}^{2}-\kappa_{n m}^{2}} y}+R_{L m} e^{i \sqrt{\zeta_{m}^{2}-\kappa_{n m}^{2}}(y+D)}\right] } \\
& \text { for }-D / 2 \leq y \leq D / 2,
\end{aligned}
$$

and $\kappa_{n m}$ is the $x$-component wavenumber of the $n$th horizontal mode resulting from the eigenvalue equation, Eq. (9), given that we have $m$ th vertical mode excited. The normalization factor $w_{n m}$ can be found numerically by employing a series of chain rules. This involves finding the first derivatives of the reflection coefficients with respect to $k_{y}$. In addition, taking a total derivative of the eigenvalue equation, Eq. (9), with respect to the frequency $\omega$ at $k_{x}=\kappa_{n m}$ yields the group slowness of the horizontal modes $\left(d k_{x} / d \omega\right)$ and hence the group velocities. To extend the solution shown in Eq. (10a)-(10c) to $|y|>D / 2$ with the assumption of adiabatic mode propagation, one can determine the unnormalized horizontal mode function outside the bounded domain by satisfying the continuity and smoothness conditions across all of the horizontal interfaces. For the horizontal ducted modes of interest, the horizontal trapped mode functions are sinusoids within the bounded domain and decay exponentially to vanish outward in the $y$ direction. Note that, in the complex contour integration, without compensation of the branch-line integral for the exponential growth of the horizontal leaky modes in the $y$ direction, the solution does not converge along the $y$-axis when the $x$-coordinate of a calculation point is close to the source. However, the boundary of this diverging area gets further and further out in the $y$ direction as the $x$-coordinate of the calculation point moves away from the source, and so the branch-line integral can generally be neglected when the computation domain of interest expands reasonably in the $x$ direction. After all, in the domain of small $x$ and large $y$ the sound field is dominated by the mode coupling effect occurring as the sound penetrates the internal waves at high grazing angles. This coupling is beyond the limitation of our 3D normal mode theory, so the branch-line integral failure is inconsequential.

\section{Radiation field}

The contribution of each vertical mode to the sound field at the termination of the duct $(x=L)$ can now be computed from Eq. (10a) $-(10 \mathrm{c})$, and then used to obtain the radiation field from the termination by adopting Huygens' principle. Under the prior assumptions, the ducted sound will pass through the termination and radiate outward, with vertical modal energy staying in the same mode (adiabatic mode propagation). Hence, the total radiation field can be obtained by summing up all the vertical mode radiations. 
The amplitude of the $m$ th vertical mode at every termination position $(x=L)$ is given by Eq. (10a)-(10c). According to Huygens' principle, solutions at $(x>L)$ must be consistent with an infinite number of "modal" point sources adjoining each other across the termination, radiating the modal energy outward. Mathematically, the solution is found using the Green's function method. The required Green's function $\mathcal{G}_{m}(x, y ; \xi, \eta)$, where $(\xi, \eta)$ indicates the termination position, i.e., $\xi=L$, is symmetric and equals $\mathcal{G}_{m}(\xi, \eta ; x, y)$ by the principle of reciprocity. This is governed by

$$
\begin{aligned}
& \left(\frac{\partial^{2}}{\partial \xi^{2}}+\frac{\partial^{2}}{\partial \eta^{2}}\right) \mathcal{G}_{m}(\xi, \eta ; x, y)+\zeta_{m}^{2} \mathcal{G}_{m}(\xi, \eta ; x, y) \\
& \quad=-\delta(\xi-x, \eta-y) .
\end{aligned}
$$

Note that since the $m$ th vertical modal amplitude at the termination provides a Dirichlet boundary condition for the radiation field, a homogeneous Dirichlet boundary condition for the Green's function $\left(\mathcal{G}_{m}(\xi, \eta ; x, y)=0\right)$ is required at $\xi$ $=L$. The method of images yields

$$
\begin{aligned}
\mathcal{G}_{m}(x, y ; \xi, \eta)= & \frac{i}{4} H_{0}^{(1)}\left(\zeta_{m} \sqrt{(\xi-x)^{2}+(\eta-y)^{2}}\right) \\
& -\frac{i}{4} H_{0}^{(1)}\left(\zeta_{m} \sqrt{(\xi+x-2 L)^{2}+(\eta-y)^{2}}\right)
\end{aligned}
$$

where $H_{0}^{(1)}$ is the zeroth order Hankel function of the first kind. With this Green's function, it is straightforward to obtain the radiated mode amplitude at $x>L$, i.e., $\Gamma_{m}(x, y)$ $=\int \Gamma_{m}(L, \eta) \partial \mathcal{G}_{m}(x, y ; \xi, \eta) /\left.\partial \xi\right|_{\xi=L} d \eta$, and $\Gamma_{m}(L, \eta)$ is the $m$ th vertical modal amplitude across the termination. Substituting the necessary terms yields

$$
\begin{aligned}
\Gamma_{m}(x, y)= & -\pi \frac{\Psi_{m}\left(x_{s}, y_{s}, z_{s}\right)}{\rho\left(z_{s}\right)} \zeta_{m} \sum_{n} \frac{\Phi_{n m}\left(y_{s}\right)}{\kappa_{n m} w_{n m}} e^{i \kappa_{n m}\left(L-x_{s}\right)} \\
& \times \int_{-\infty}^{\infty} \Phi_{n m}(\eta) \frac{x-L}{\sqrt{(x-L)^{2}+(y-\eta)^{2}}} \\
& \times H_{1}^{(1)}\left(\zeta_{m} \sqrt{(x-L)^{2}+(y-\eta)^{2}}\right) d \eta,
\end{aligned}
$$

where $H_{1}^{(1)}$ is the first order Hankel function of the first kind, resulting from differentiation of $H_{0}^{(1)}$. It is numerically straightforward to implement this Hankel integral, which is done in Sec. II C. Alternatively, with the assistance of the principal asymptotic form of the Hankel function for large arguments, the far-field approximation is given by

$$
\begin{aligned}
\Gamma_{m}(x, y)= & 2 \pi e^{i(\pi / 4)} \sqrt{\zeta_{m}} \frac{\Psi_{m}\left(x_{s}, y_{s}, z_{s}\right)}{\rho\left(z_{s}\right)} \\
& \times \sum_{n} \frac{\Phi_{n m}\left(y_{s}\right)}{\kappa_{n m} w_{n m}} e^{i \kappa_{n m}\left(L-x_{s}\right)} \Omega_{n m}(r, \theta),
\end{aligned}
$$

where $\quad r=\sqrt{(x-L)^{2}+y^{2}}, \quad \theta=\tan ^{-1}[y /(x-L)], \quad \Omega_{n m}(r, \theta)$ $=r^{-1 / 2} e^{i \zeta_{m} r} \sin \theta \hat{\Phi}_{n m}(\theta)$, and $\quad \hat{\Phi}_{n m}(\theta)=(2 \pi)^{-1 / 2} \int_{-\infty}^{\infty} \Phi_{n m}(\eta)$ $\times e^{-i 2 \pi u \eta} d \eta$, where the $u$ in the exponent equals $\zeta_{m} \sin \theta /(2 \pi)$.
The normal mode solutions for the ducted field and the radiation field have been obtained, and now we shall re-visit the prior assumption of total transmission at the open-ended termination. Remember that since the termination gap is very large compared to the underwater acoustic wavelength of interest in a common case, the $k a$ value (acoustic wavenumber multiplying the gap aperture) is large and thus only tiny amount of reflection energy is yielded, which means that the assumption of total transmission should be valid. In addition, we can examine the continuity of the normal derivative of the pressure field solution (the smoothness) across the termination, as we notice there is only pressure continuity condition being used in deriving the radiation field solution. If the assumption of total transmission is sustained, the pressure field solution should be smooth across the termination, as shown in the next computational example.

\section{Computational example}

A numerical integration scheme is utilized to compute the analytic solution shown in Eq. (13), and the environmental model considered here is illustrated in Fig. 1(a). To remind the reader, a 3D Cartesian coordinate system $(x-y-z)$ is chosen for positioning (see the figure for the orientation). The water column has two homogeneous layers; the sound speed in the upper layer is $1520 \mathrm{~m} / \mathrm{s}$, faster than the lower layer, where the sound speed is $1480 \mathrm{~m} / \mathrm{s}$. The density in the water column (in both layers) is $1.0 \mathrm{~g} / \mathrm{cm}^{3}$. The water depth is $80 \mathrm{~m}$, and the thickness of the upper water layer is $20 \mathrm{~m}$. Two nonlinear internal waves with square waveform disturb the water column, and their wavefronts are both parallel to the $x$-axis. These internal square waves have the same amplitude, $20 \mathrm{~m}$, and wavelength, $200 \mathrm{~m}$, and they both abruptly terminate at $x=20 \mathrm{~km}$. The gap between the internal waves is $300 \mathrm{~m}$ wide. The bottom is considered to be homogeneous, with sound speed of $1700 \mathrm{~m} / \mathrm{s}$, sound attenuation coefficient of $0.5 \mathrm{~dB} / \lambda$, and density of $1.5 \mathrm{~g} / \mathrm{cm}^{3}$.

The first result shown here is the dependence of the radiation patterns of the vertical modes on the acoustic source position. A sinusoidal $100-\mathrm{Hz}$ source is considered, and Fig. 2 shows the intensity contours of the first and second vertical modes excited by the source located at four different $x-y$ positions in the gap between nonlinear internal waves. The internal waves form a horizontal duct at positions $0 \leq x \leq 20 \mathrm{~km}$ where the sound transmitted from the source is trapped. The waves terminate at $x=20 \mathrm{~km}$, and the trapped sound radiates away at this position. Note that the source depth is fixed $\left(z_{s}=-70 \mathrm{~m}\right)$, and the internal waves have a square shape so that excitation of vertical modes is the same for each of the four source positions. There are more than two vertical modes excited by the source, but only the first two of them are shown in the plot. As shown in the figure, each vertical mode has its unique radiation pattern. That is because the across-duct energy distribution of each vertical mode at the termination is unique [see Eq. (13)]. Note also that the radiation pattern of each vertical mode differs dramatically for different source positions in the duct between the waves. The reason is that for a given vertical mode the excitation of the horizontal modes in the duct strongly de- 

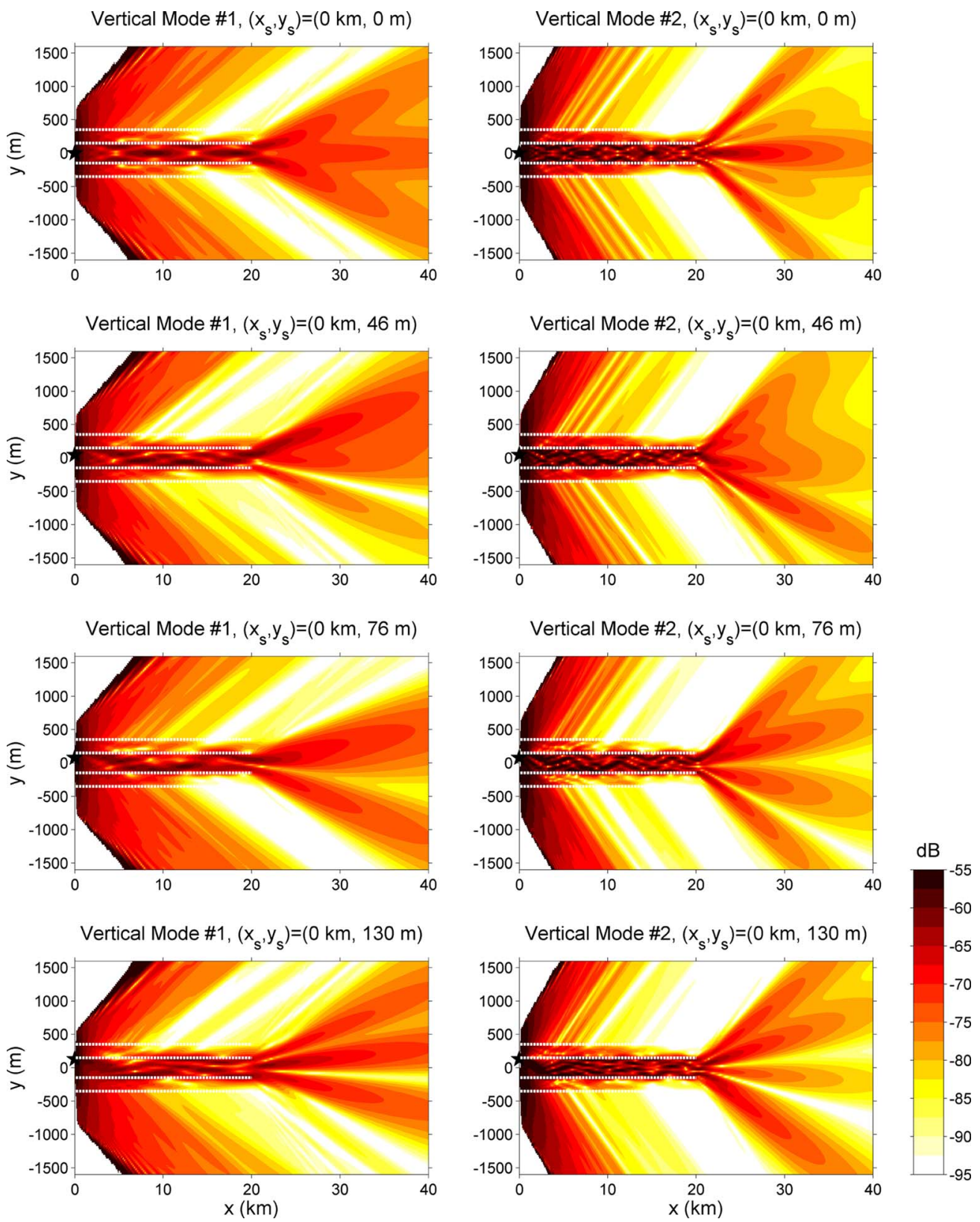

FIG. 2. (Color online) Intensity is shown for the first two vertical modes excited by a sinusoidal $100-\mathrm{Hz}$ source located at four different $y$ positions (shown from top to bottom). The source is always in the gap between the nonlinear internal waves, and the simplified model of Fig. 1 is used. Mode 1 intensity is shown on the left, mode 2 on the right. The source depth is fixed. The edges of internal waves are indicated by dashed lines. See the main text for the detailed environmental and acoustic source parameters.

pends on the source position in the $y$-axis [see Eq. (10a)-(10c)], so the across-duct energy distribution of the given vertical mode at the termination is a function of source position, and thus the radiation pattern is a function of source position.

The next computational result shows broadband sound radiation from the termination of an identical truncated internal square-wave duct. The previous harmonic source is replaced by a source emitting a broadband signal which contains 16 periods of a $100-\mathrm{Hz}$ sine wave and is tapered by the Hann window. Note that $99.95 \%$ of the source energy is contained in the $25-\mathrm{Hz}$ bandwidth centered at $100 \mathrm{~Hz}$. This broadband source is fixed at a position of $\left(x_{s}, y_{s}, z_{s}\right)$ $=(0 \mathrm{~km}, 0 \mathrm{~m},-70 \mathrm{~m})$, and a hydrophone array is placed at $x=30 \mathrm{~km}(10 \mathrm{~km}$ distant from the termination of the internal-wave duct). The array has a vertical component [vertical line array (VLA)] covering the whole water column and located at $y=0 \mathrm{~m}$ and a horizontal component [horizontal line array (HLA)] placed on the bottom and extending along the negative $y$-axis for $1000 \mathrm{~m}$.

The internal waves form a horizontal acoustic duct at positions $0 \leq x \leq 20 \mathrm{~km}$. Horizontal modal dispersion in the duct is examined here because this phenomenon affects the broadband result. The dispersion of horizontal modes can be quantified by modal group velocities. Figure 3 shows how, for this duct configuration, the group velocities of the horizontal modes associated with the second vertical mode vary with the horizontal mode number and the frequency. The group velocities of horizontal modes have upper limits given by the group velocity computed using profiles in the wave 


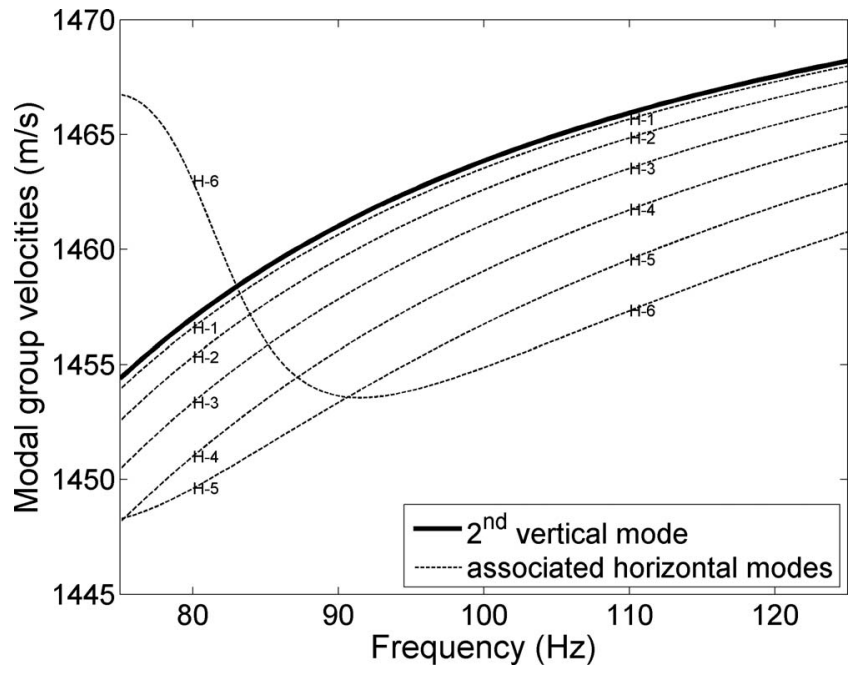

FIG. 3. Group velocities of the unperturbed second vertical mode (solid line) and six of its associated horizontal ducting modes (dashed lines) are shown for the simplified model of Fig. 1. The six horizontal modes are labeled by numbers.

crests (i.e., away from the wave duct region, which happens to be the profile in the duct itself for this idealized configuration). However, when the horizontal mode angle with respect to the internal-wave crests, determined from $\cos ^{-1}\left(\kappa_{n m} / \zeta_{m}\right)$, nears the critical grazing angle for total internal reflection, the horizontal modal energy tunnels through the internal-wave trough, where the vertical modal phase and group velocities are higher, and thus the horizontal modal group velocity increases and exceeds that limit (see the sixth horizontal mode in Fig. 3).

The four panels in Fig. 4 illustrate the magnitude of the broadband acoustic time series received on the hydrophone arrays (both on the VLA and the HLA) when the truncated internal-wave duct is located at four different positions along the $y$-axis. As shown in the plot, each vertical mode arrival is well separated in time at the array; the arrivals on the VLA nicely represent the vertical mode shapes, and the arrivals on the HLA provide a means for us to observe the energy distribution of each vertical mode along the $y$-axis. Due to the change in source position in the internal-wave duct, modal radiation patterns at these four cases are different, and we do see the intensity of modal arrivals changing. This broadband source calculation does show the effect of horizontal modal dispersion (see the multiple arrivals of the second vertical mode in the top two panels of the plot). This is indeed a nice illustration in the time domain that the modal field now has vertical and horizontal mode numbers.

\section{3D PARABOLIC EQUATION MODELING}

In the ocean, background sound speed profiles and nonlinear internal waves are not as simple as in the previous simplified model, and it would be challenging if not impossible to obtain an analytical solution for the 3D sound field. In order to handle a more realistic case, an acoustical propagation program ${ }^{10}$ using the $3 \mathrm{D}$ parabolic approximation is employed here. The modeling technique utilized in this program is discussed briefly below, and the reader is referred to

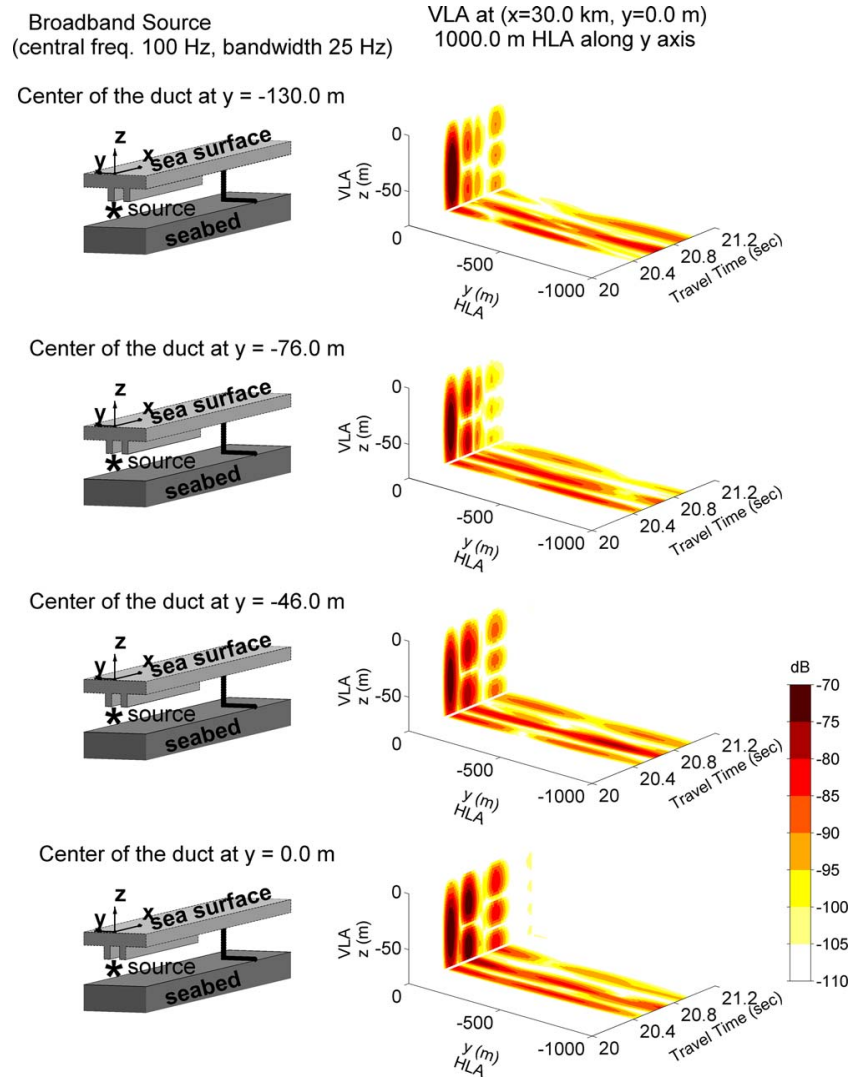

FIG. 4. (Color online) Broadband sound radiation from the termination of the internal-wave duct in the simplified mode. From top to bottom, the $y$ position of the source with respect to the waves differs. The right panels illustrate the pulse intensity received at the horizontal and vertical arrays, denoting by thick lines in the left panels. See the main text for the detailed information about the model parameters.

the technical report ${ }^{10}$ for further details. Note that the parabolic approximation is more complete than the approach of Sec. II because it allows mode coupling, which has been observed to occur in the field, but is not expected to occur near the termination which we are modeling.

\section{A. Split-step Fourier algorithm}

The acoustical propagation program we use employs the split-step Fourier (SSF) technique ${ }^{17}$ to solve the 3D parabolic acoustic wave equation (PE) for one-way propagating waves from a harmonic source in a Cartesian coordinate system. The SSF technique divides propagation over each distance increment through a heterogeneous sound speed environment into step-by-step "free space" propagation through a medium having a fixed reference wavenumber and periodically introduced (at each step) phase fluctuations consistent with departures from that fixed speed. The free space propagation is handled in the wavenumber domain, and the phase anomalies are introduced in the spatial domain. Amplitude effects such as absorption are introduced with the phase anomalies. Thus, each step (defined to be in the $x$ direction) requires a $2 \mathrm{D}$ Fourier transform and an inverse 2D Fourier transform. Note that the wide-angle variant of the propagation operator ${ }^{18}$ is used. 


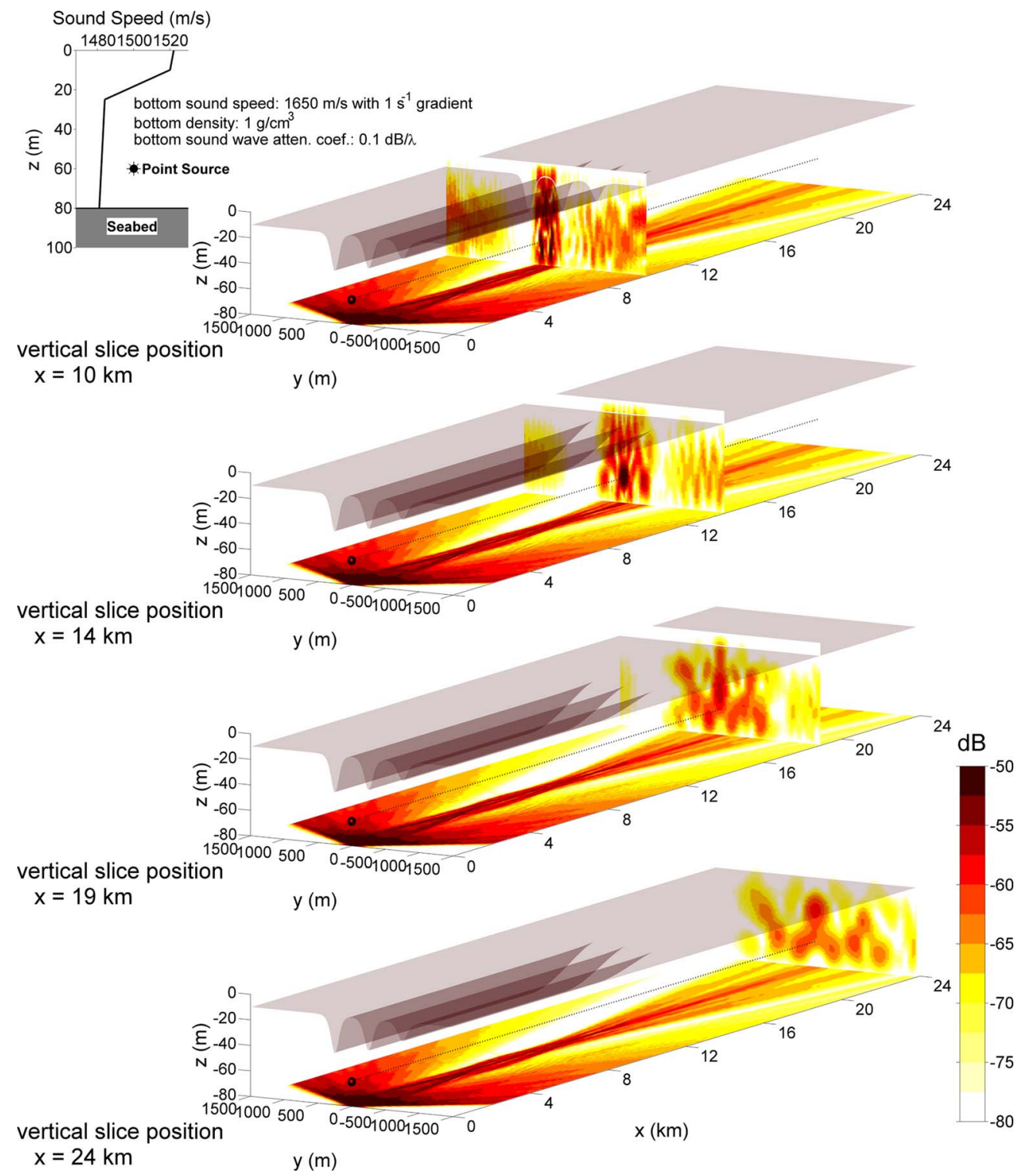

FIG. 5. (Color online) Numerical simulation of 3D sound propagation in an internal-wave duct model. (a) Upper left panel: environmental conditions in the numerical calculation. The sound speed profile represents a downward refracting situation in shallow water ocean. A sinusoidal 200-Hz source is located at 60-m depth. (b) Lower right panel: illustrations of 3D sound pressure fields. On the bottom of the visualization volume a 2D distribution of depth-averaged sound intensity is shown. The vertical slices show intensity on the slice. The transparent surface illustrates the displacement of the top of thermocline layer, i.e., internal waves.

\section{B. Numerical simulation example}

The environmental conditions for the numerical simulation using the Cartesian 3D PE program are illustrated in the upper-left panel of Fig. 5 and are described here. The water depth is $80 \mathrm{~m}$, and a typical downward refracting sound speed profile observed in the ocean is considered. The bottom geoacoustic properties are listed in the figure. Note that the bottom density is set to be $1 \mathrm{~g} / \mathrm{cm}^{3}$ because the current version of this Cartesian 3D PE program does not allow medium density to vary; however, the conclusion drawn from the simulation result should still be valid. The nonlinear internal-wave chain moving in the $y$ direction consists of three solitons, which depress the thermocline into the deeper water column. Although the solitons have different amplitudes, they all satisfy the Korteweg-de Vries (KdV) nonlinear internal-wave equation. Along the soliton wavefront (see the transparent surface shown in the lower-right panels of Fig. 5), the internal-wave waveform extends to $x=10 \mathrm{~km}$ distant from the acoustic source with a permanent shape and then gradually diminishes to zero within a $4 \mathrm{~km}$ distance (14 km total).

The first numerical calculation is for narrowband sound propagation. The acoustic source, emitting $200-\mathrm{Hz}$ sound, is placed between the biggest two solitons and at $60-\mathrm{m}$ depth in the water, close to a node of the third acoustic mode. The calculation domain of the 3D PE program is configured in such a way that we can simulate sound propagating in the nonlinear internal-wave duct and radiating outward from the termination of the duct. A mode filtering is employed to obtain acoustic mode amplitudes at each $(x, y)$ position from the full-field solution. The 3D visualization volume in Fig. 5 shows the simulation results. On the bottom of the visualiza- 

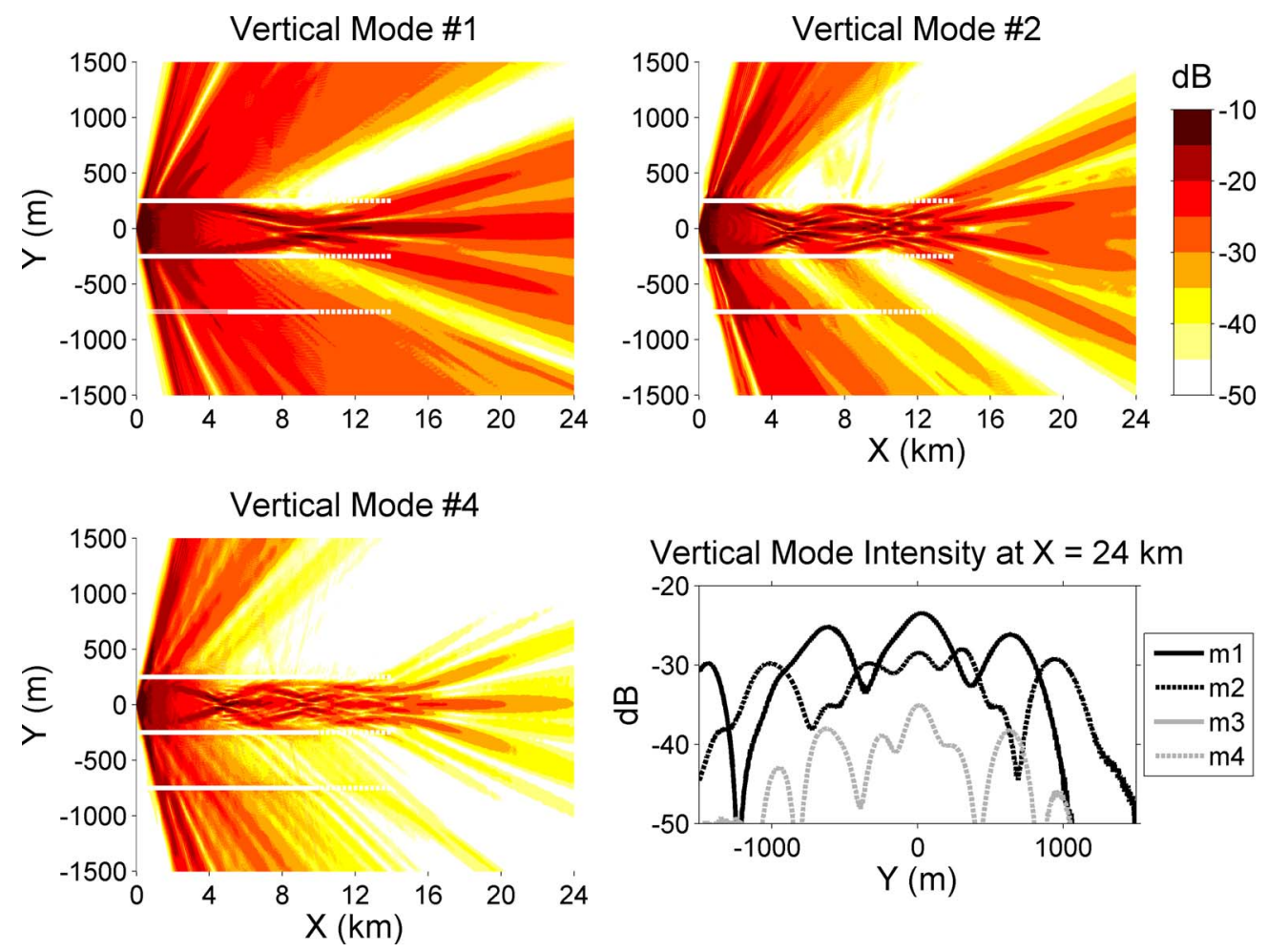

FIG. 6. (Color online) Vertical mode intensity is shown for propagation in the environmental model of Fig. 5. The solid lines are along internal-wave troughs, and the dashed lines indicate the "diminishing region" of the internal waves. See the main text for detailed discussions. At the lower right, the modal intensity at the right hand edge of other panels is plotted.

tion volume a $2 \mathrm{D}$ distribution of depth-averaged sound intensity is presented, and a vertical slice slides through the field to show detailed 3D structure. The mode amplitudes resulting from mode filtering are shown in Fig. 6 as horizontal contours, from which the following is observed.

Within the first $10-\mathrm{km}$ propagation distance, despite some of the sound energy escaping at high angles with respect to the duct direction, the modal interference process yields a strong focusing effect in the internal-wave duct, which is consistent with what has been reported in the literature. ${ }^{1,3,5-7}$ The radiation beam pattern, unique to each mode, starts to develop in the diminishing region of internal waves and becomes fully developed after the sound exits the duct with the features suggested by the simplified analytic model in Sec. II. Note that from the plot of mode amplitudes (Fig. 6) no mode-coupling effect is observed in both the ducted sound area and the radiation field, which confirms the assumption of adiabatic mode propagation used in deriving the normal mode solution.

The next case is broadband sound propagation, and the previous narrowband source is replaced by a source emitting a broadband signal which contains 16 periods of a $200-\mathrm{Hz}$ sine wave and is tapered by a Hann window. Since $99.95 \%$ of the source energy is contained in the $50-\mathrm{Hz}$ band centered at $200 \mathrm{~Hz}$, one can calculate the fields for frequencies from 175 to $225 \mathrm{~Hz}$ (at $N-\mathrm{Hz}$ spacing) and sum them to produce a $N^{-1}$-second-long broadband reception at any receiving point. The four panels in Fig. 7 illustrate the magnitude of the broadband signals received at four VLAs distributed across the wavefront at the end of the propagation distance $(X$ $=24 \mathrm{~km})$. The VLA locations are chosen to reveal the spatial variability of broadband sound radiation from the open end of the nonlinear internal-wave duct. A good reference VLA is the one located at the source axis (labeled as $Y=0 \mathrm{~m}$ ), where all vertical modes except for the third mode are distinct. Note that since the source is placed close to a depth null of the third mode, insignificant modal energy is excited. First we see that at $Y=300 \mathrm{~m}$ the fifth mode fades out, and (more dramatically) at $Y=1101 \mathrm{~m}$ only the second mode is observable. In addition, as seen in the previous analytical compu-
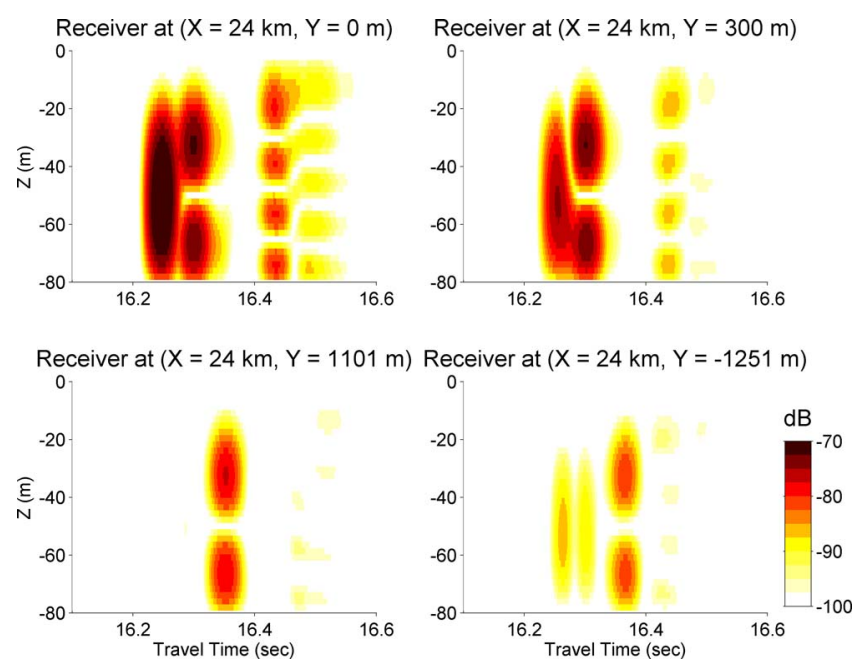

FIG. 7. (Color online) Simulation results for broadband sound $(50-\mathrm{Hz}$ bandwidth centered at 200-Hz) propagation in the environmental model of Fig. 5. These four panels show the intensity of the broadband signals received at four vertical hydrophone line arrays distributed across the wavefront at $X$ $=24 \mathrm{~km}$. 


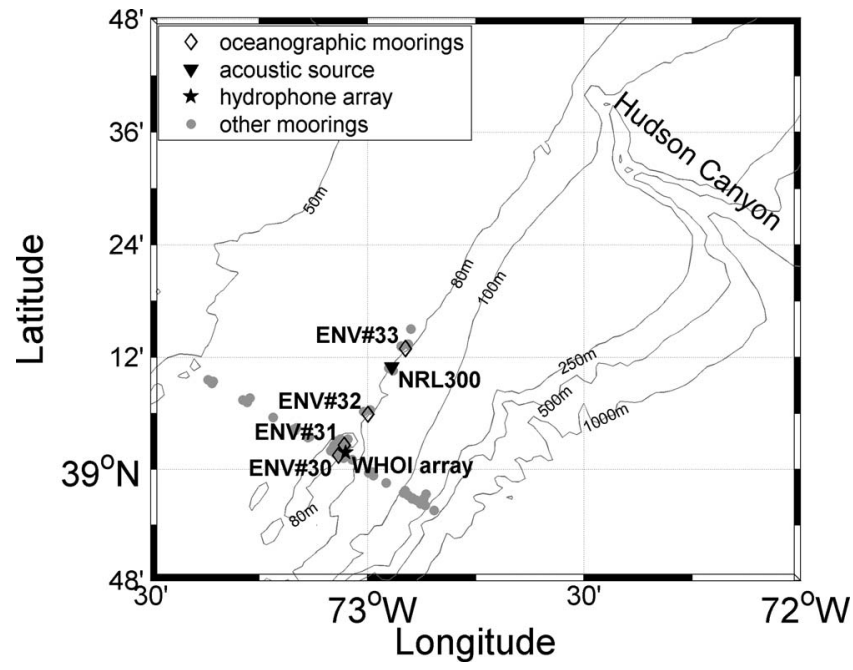

FIG. 8. Study area of the SW06 experiment. The depth of each isobath line is labeled. A total of 62 moorings was deployed in a " $T$ " geometry to create an along-shelf path and an across-shelf path. The data collected from the six labeled moorings on the along-shelf path are used in this paper.

tation, multiple arrival of the same mode is also observed in this numerical calculation (mode 1 at $Y=-1251 \mathrm{~m}$ ). All of these plots truly convey the complexity of the sound field due to the modal radiation effect.

\section{EXPERIMENTAL DATA}

In Secs. II and III theoretical and numerical analyses have been implemented to study acoustic mode radiation from the terminations of idealized square-wave and $\mathrm{KdV}$ model internal-wave ducts. Here we shall examine acoustic signals measured in the coastal ocean for evidence of features similar to those seen in the computational examples. In the summer of 2006 a large multi-disciplinary experiment, SW06, ${ }^{11}$ was conducted on the Mid-Atlantic Bight continental shelf at a location about $160 \mathrm{~km}$ east of the New Jersey coast and about $80 \mathrm{~km}$ southwest of the Hudson Canyon (Fig. 8). A total of 62 acoustic and oceanographic moorings was deployed in a "T" geometry to create an along-shelf track (following the 80-m isobath line) and an across-shelf track (depths changing from 50 to $500 \mathrm{~m}$ ). In this paper, we will focus on the data collected from some of the moorings deployed on the along-shelf track. At the southern end of the along-shelf track, an underwater hydrophone-array system (labeled by "WHOI array" as it was deployed by the acoustics group from the Woods Hole Oceanographic Institution) was positioned. This system has two arrays: one is a 16channel VLA covering the water column from depth $13.5 \mathrm{~m}$ to the bottom, and the other is a 465-m-long HLA with 32 uniformly-spaced hydrophones deployed on the bottom in a strict N-S orientation. There were many acoustic sources deployed in the water during the SW06 experiment, and the specific source of interest on the along-shelf track is a source transmitting a frequency modulated (FM) sweep sound centered at frequency of $300 \mathrm{~Hz}$ with $60-\mathrm{Hz}$ bandwidth (labeled by "NRL300" as this source is owned by the U.S. Naval Research Laboratory). Water temperature data collected at the NRL300 source mooring and the WHOI array will be used to identify internal waves. Four oceanographic moorings deployed on the along-shelf track (labeled by ENV\#3033) also provide other useful environmental measurements, including water temperature and current speeds.

The environmental data indicate that a strong nonlinear internal-wave packet with a somewhat straight wavefront started passing through the along-shelf mooring track at about 10:00 Greenwich mean time (GMT) on August 20 (see Fig. 9). The angle between the wavefront and the along-shelf track was about $6 \mathrm{deg}$. The environmental data also indicate that this internal-wave packet terminated between moorings ENV\#32 and ENV\#31, and that the exact terminating point should be around $13 \mathrm{~km}$ distant from the NRL300 source and $5 \mathrm{~km}$ distant from the WHOI array. The NRL300 source was designed to transmit sound for $7.5 \mathrm{~min}$ at every hour and half-hour. At the next transmission (10:30 GMT) after the internal-wave packet reached the NRL300 source, the source was still in the packet. Hence it can be expected that the sound transmitted from the source would be trapped in the duct formed by the internal waves and propagate toward the termination of the internal-wave packet between moorings ENV\#32 and ENV\#31. The trapped sound would then radiate out from the termination and finally reach the WHOI array. In the following, we will see that the sound field received at the array was structured in a way that suggests that ducting and radiation from the duct had strong influences.

The NRL300 source linearly swept over $270-330 \mathrm{~Hz}$ in exactly $2.048 \mathrm{~s}$ every $4.096 \mathrm{~s}$ for 110 times, which yielded a 7.5-min-long transmission, at every hour and half-hour. Each sweep was tapered with a 0.2048 -s amplitude taper $(10 \%$ cosine taper) at the beginning and the end to allow gradually ramping on and off. A standard matched filter can compress the sweep to $33 \mathrm{~ms}$, but with significant side-lobe ripples that could mask the multi-modal arrivals in this case. To reduce the side lobes, one can apply a taper window on the replica waveform of the matched filter ${ }^{19}$ at the cost of increasing the (compressed) pulse length. In this data analysis the Hamming window function is applied, and the matched filter with such a tapered replica waveform produces 66-ms-long compressed pulses with side lobes less than $-30 \mathrm{~dB}$. Three of the 110 pulses are shown in Fig. 10, where one can clearly see complicated temporal and spatial variability. To further analyze the data and quantify the variability, the following modal analyses are performed.

Modal excitation by the NRL300 source during the transmission period encountering the truncated internal-wave packet is calculated by the acoustic normal mode program KRAKEN. $^{20}$ The in situ water sound speed measurements on the NRL300 source mooring and a bottom model provided by a previous study ${ }^{21}$ are used in the program. The calculation results, shown in Fig. 11, indicate that the variation in modal energy excitation caused by the local water-column fluctuations is less than $3 \mathrm{~dB}$, with the maximum variation occurring in the first mode. A series of broadband mode filterings is performed next to examine the vertical modal content of the received pulses on the WHOI VLA. The vertical mode functions used in the filter are calculated by the KRAKEN program, and the in situ water sound speed measurements on the WHOI VLA and the same bottom model 

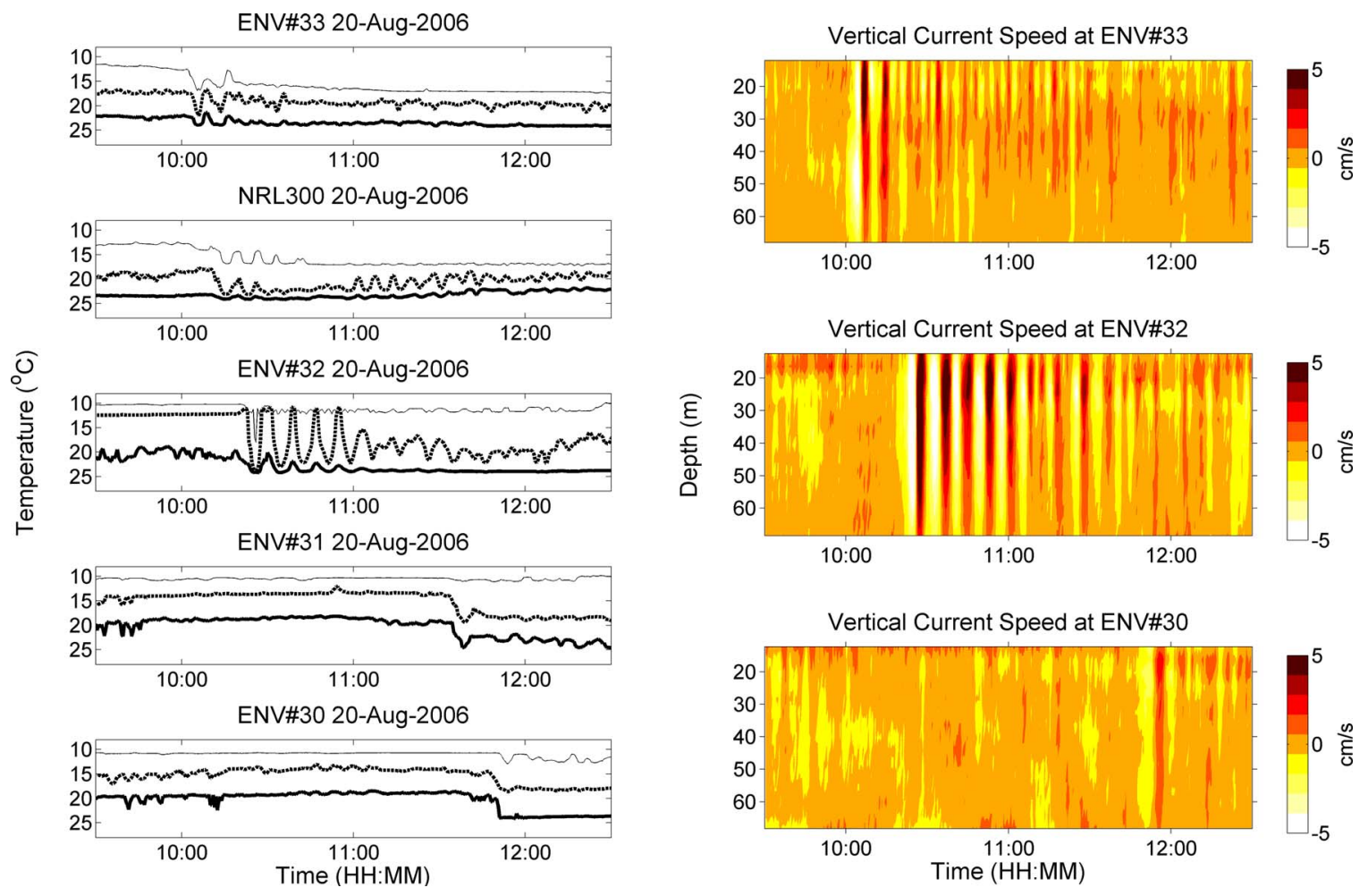

FIG. 9. (Color online) Environmental data collected from the moorings distributed along the 80-m-isobath track (total $30 \mathrm{~km}$ long) in the SW06 experiment on August 20 from 8:00 to 14:00 (GMT). Left panels: temperature data at three different depths about $10 \mathrm{~m}$ (thick line), $20 \mathrm{~m}$ (dashed line), and $30 \mathrm{~m}$ (thin line). Right panels: vertical current speeds measured by the acoustic Doppler current profilers. Both temperature and current speed data show that a truncated nonlinear internal-wave packet, terminating between moorings ENV\#32 and ENV\#31, passed through the mooring track.

for modal excitation calculation are used. Figure 12 shows the vertical mode functions of frequency $300 \mathrm{~Hz}$ at 10:32:30 GMT. The mode-filter outputs, shown in Fig. 13, reveal a very interesting mode-dependent variation pattern; one can see that modal arrival levels can vary up to $30 \mathrm{~dB}$ in $3 \mathrm{~min}$, and also that modal arrivals alternately diminish. This is quite striking. Examining possible causes of this, given the good coverage of the WHOI VLA in the water column (see Fig. 12), there should not be a serious modal cross-talking defect in the mode filtering process, especially for the lowest three modes. Hence the modal variation seen in the filter outputs is not due to imperfect signal processing. Another possible explanation is variable modal excitation. A modal excitation calculation suggests that less than $3-\mathrm{dB}$ variation should be expected, which cannot explain the $30-\mathrm{dB}$ variations in modal arrival levels, not even if one placed considerable uncertainty on the modal excitation prediction. Invoking known mechanisms for mode coupling is also unreasonable because the fairly straight wavefront of the truncated internal-wave packet is only about $6 \mathrm{deg}$ to the propagation path from the source to the receiver, which is smaller than the typical angles causing coupling (although the very largest waves measured in the SW06 area, 20-m amplitude, may cause coupling at $8 \mathrm{deg}^{22}$ ). Thus, the modal arrivals are most consistent with time-variable of acoustic mode radiation from the internal-wave duct termination. As the internal-wave duct passed over the NRL300 source the relative source position in the duct changed, causing horizontal modal radiation pattern fluctuation in the manner illustrated by the examples.

\section{DISCUSSIONS}

The theoretical and numerical calculations clearly show that variable acoustic mode radiation from the termination of an internal-wave duct can be a significant source of spatial and temporal fluctuations of sound outside the duct. The spatial fluctuations result from the narrow angular extent of the radiation beams, for each mode, that emerge from the termination. One consequence is that the sound intensity in the areas not covered by radiation beams is reduced. The beams have temporal variability because passage of a truncated internal-wave duct over a fixed source causes the source position in the duct to vary in time, thus giving a timedependence to the excitation of horizontal mode and thus to the emerging beams.

The real oceanic environment is often more complicated than the modeled environments used in this paper, and there are other acoustic effects that cause acoustic fluctuation patterns that are similar to the modal beam effect and that we shall be aware of. For instance, the effect of mode coupling on changing received modal intensity can be similar to the modal beam effect. To distinguish these two effects, one may need to carefully examine environmental data and determine which effect is more likely to occur in the environmental condition, as done here for the NRL300 signals. The following is another example. The calculations herein show that horizontal modal dispersion within the duct may cause multiple arrival of a mode (see the top two panels of Fig. 4 and the lower right panel of Fig. 7). However, in sound transmission data collected in the 1988 Hudson Canyon 


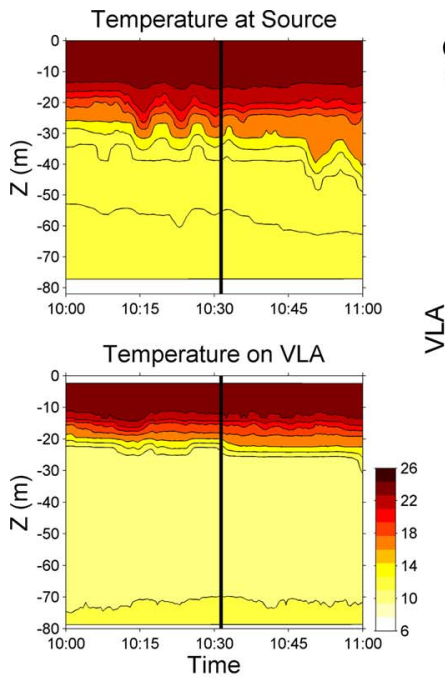

Compressed pulses received at the WHOI array in the SWO6 experiment
at 10:31:24 on Aug-20 in 2006 (an LFM source, $f=300 \mathrm{~Hz}, \mathrm{BW}=60 \mathrm{~Hz}$ )
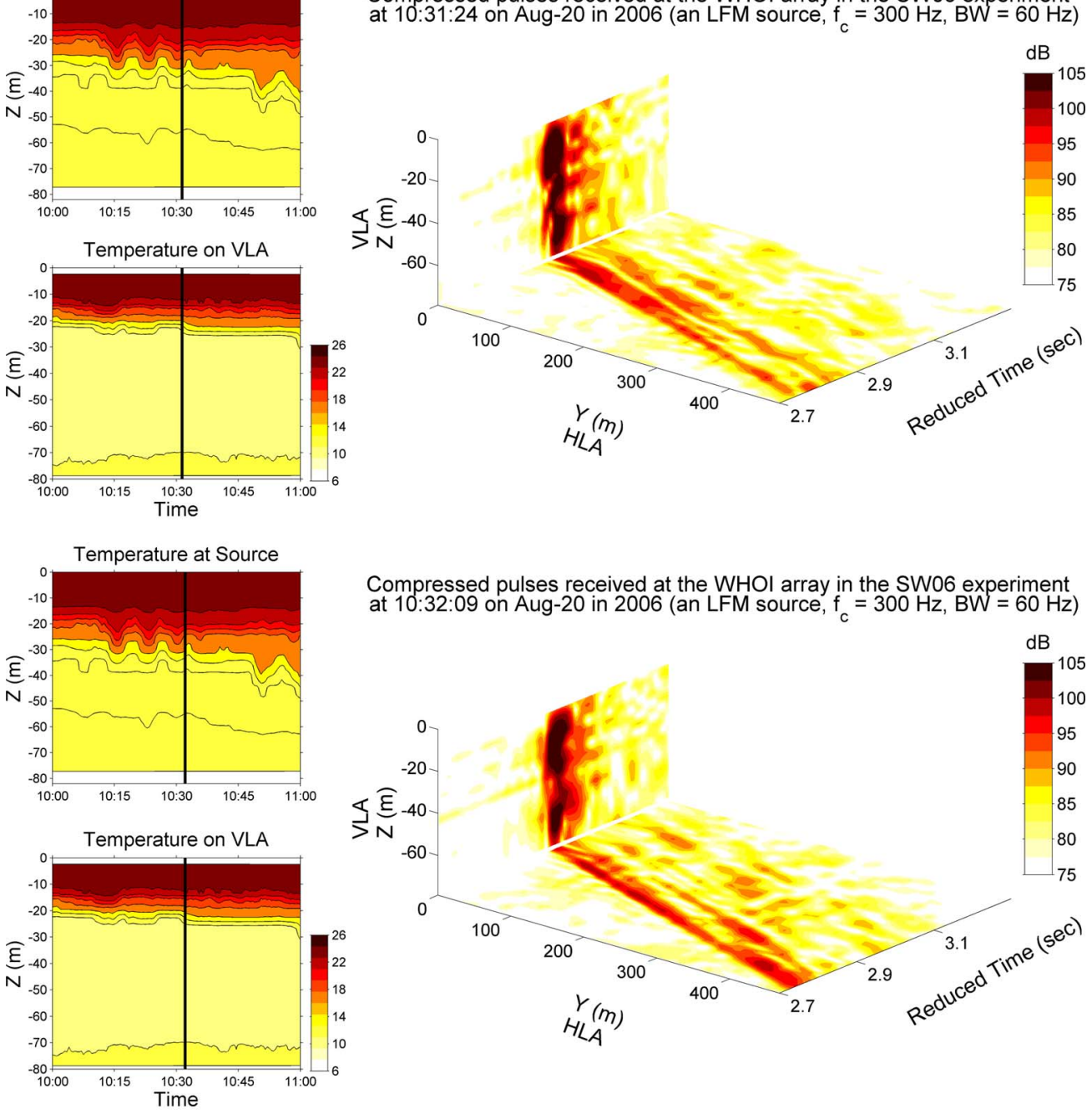

Compressed pulses received at the WHOI array in the SW06 experiment
at 10:32:09 on Aug-20 in 2006 (an LFM source, $f=300 \mathrm{~Hz}, \mathrm{BW}=60 \mathrm{~Hz}$ )
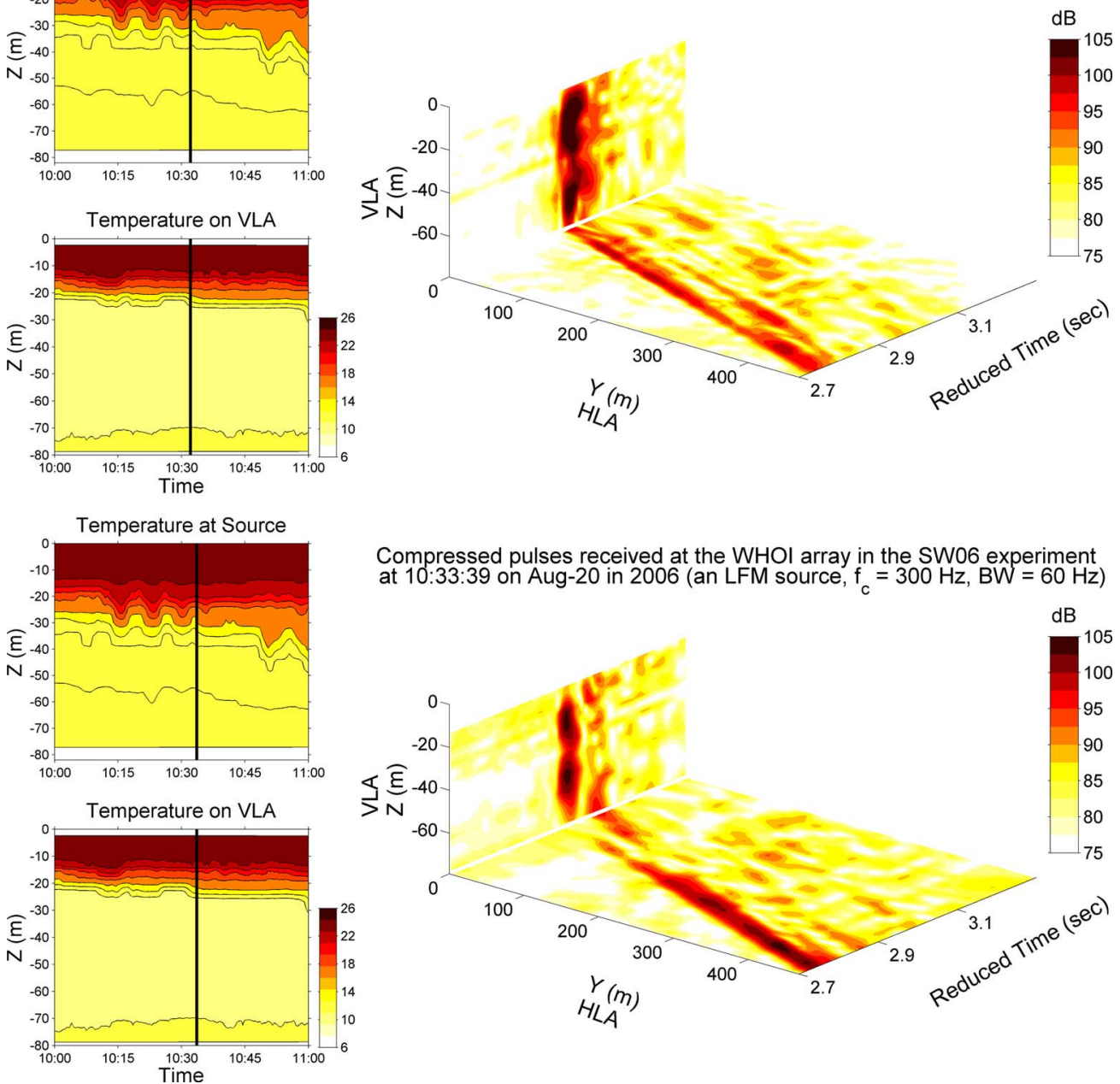

at 10:33:39 on Aug-20 in 2006 (an LFM source, $f_{c}=300 \mathrm{~Hz}, \mathrm{BW}=60 \mathrm{~Hz}$ )

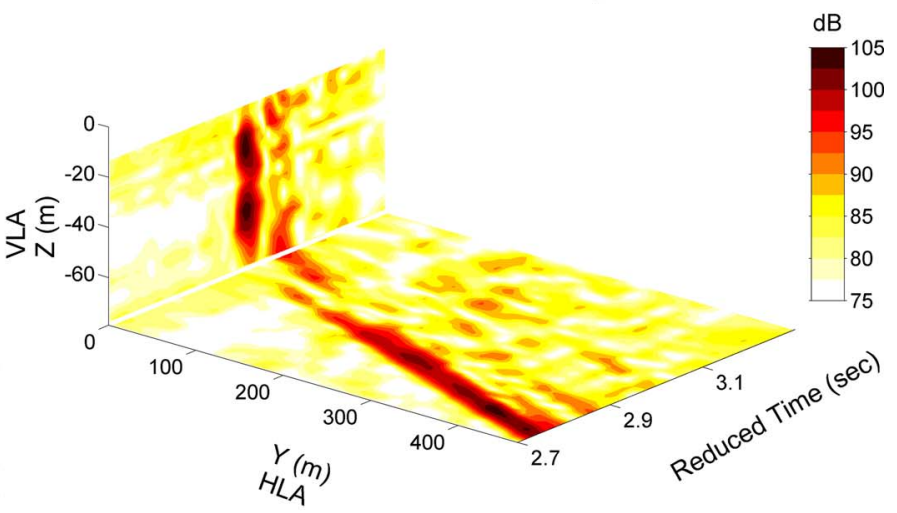

FIG. 10. (Color online) Compressed pulses of the NRL300 sweep signals received on the SW06 WHOI array. (Right) Pulse arrivals at three times are shown, from top to bottom. To the left of each pulse plot, temperature vs depth time series for the source and receiver positions are shown. The line indicates the condition at the time of transmission. At these times, the source was in the truncated internal-wave packet shown in Fig. 9.

Experiment, ${ }^{23}$ Cederberg et al. ${ }^{24}$ observed that a deep subbottom duct may produce two different modes with almost identical wavenumbers and with shapes that are similar in the water column. Separate arrival of the two modes may be mistaken for multiple arrival of the same mode, particularly when noise is present. This sub-bottom ducting effect presents an alternative explanation of the double (repeat) mode features.

During the SW06 experiment, other internal-wave ducting situations were observed, such as curved waves or frag- 


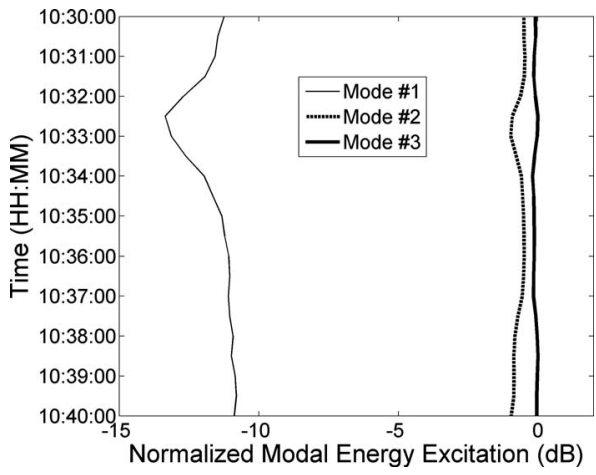

FIG. 11. Time series of theoretical estimates of the energy excitation of first three modes by the NRL300 source during the truncated internal-wave event shown in Fig. 9 on August 20.

mented waves. In the interesting curved wave situation, sound initially trapped in the duct may escape due to the change in the grazing angles of trapped sound. That is, horizontal modes that are sub-critical may become supercritical and escape. As for a fragmented internal-wave field, sound will encounter multiple processes of trapping and radiating, and the single truncation case reported in this paper may serve as a starting point toward understanding that multiplescatter case. It is also worth mentioning that when an acoustic source is just outside and close to a truncated internalwave duct, the significance of the mode radiation effect depends on how far the source is away from the duct. If it is close, a certain amount of energy may still be trapped in the duct after the sound refracts into the duct, and so at the termination one can still observe significant radiation effects.

The modal radiation may affect horizontal array beamforming. The simulations show that the termination acts like a sound projector, and horizontal beamforming may show incorrect sound source bearing directed toward the termination. Note that narrow beams are not plane waves, and standard beamforming with arrays larger than the beam width may be inconclusive. Also, because of the interference of the radiation patterns of different modes, the array coherent length in the radiation field may be much shorter than what would be expected at array locations far from internal waves

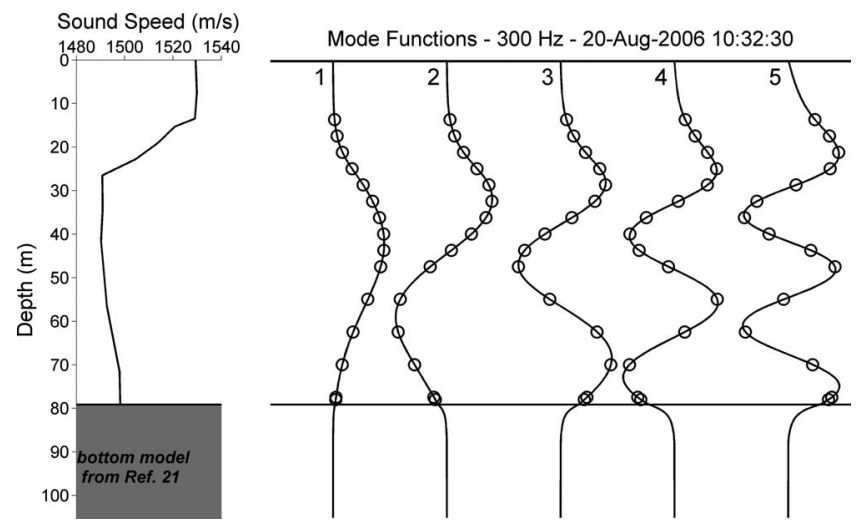

FIG. 12. Estimates of the mode functions of frequency $300 \mathrm{~Hz}$ at the location of WHOI VLA at 10:32:30 GMT on August 20 in the SW06 experiment. The in situ water-column sound speed profile and a bottom model derived from Ref. 21 are used for calculating the modes. The circles on the curves indicate the hydrophone depths on the VLA.
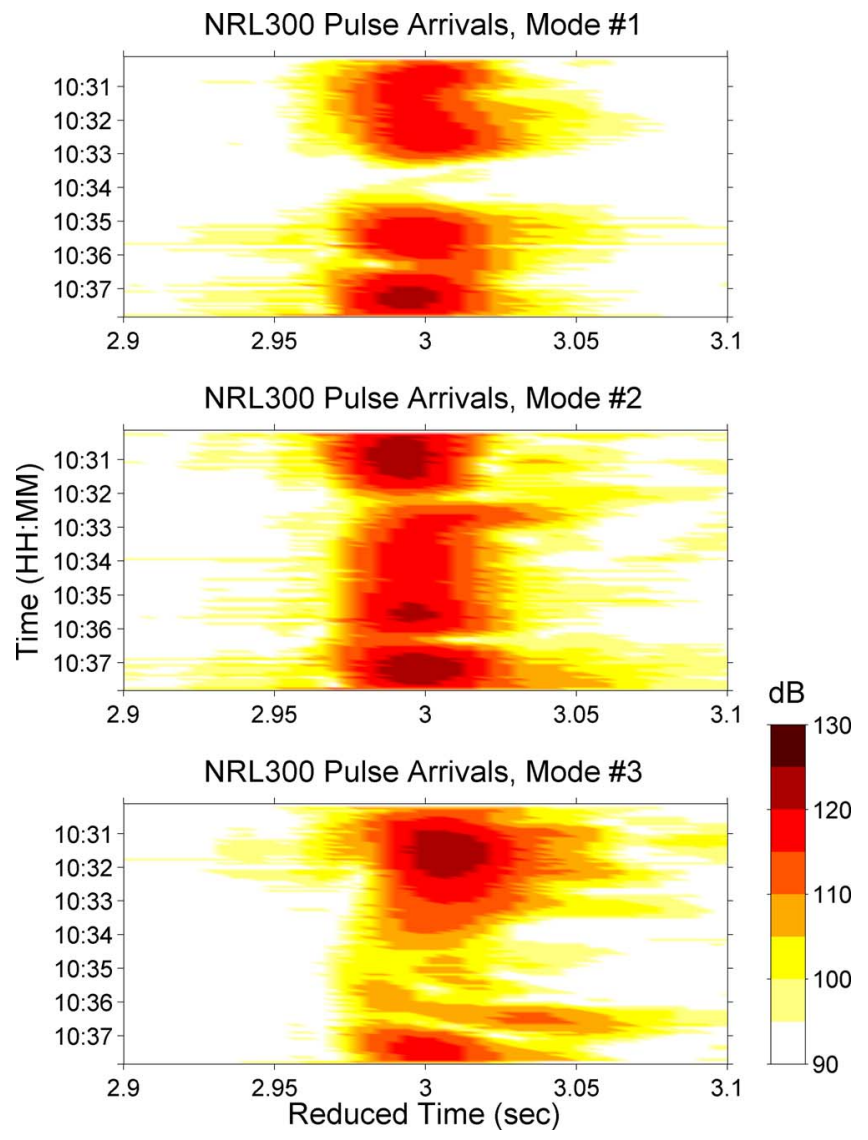

FIG. 13. (Color online) Broadband mode-filtered outputs of the received NRL300 pulses on the WHOI VLA during the truncated internal-wave event on August 20 in the SW06 experiment. The first three modes are shown, and a total of 110 pulses is analyzed. The mode intensities fluctuate in time.

(a typical value in the SW06 site is 15 wavelengths at $100 \mathrm{~Hz}$, according to the measurement of Collis et al. ${ }^{25}$ during quiescent periods. Further analysis of a larger portion of this data set shows higher values of 25-30 wavelengths during the first week of the experiment).

\section{CONCLUSIONS}

The sound radiation from the termination of a truncated internal-wave duct has been studied with both analytical and numerical approaches. A 3D normal mode solution has been derived to describe the radiation field in a simplified truncated internal-wave duct model, and a computer code implementing 3D PE approximation is employed to investigate the radiation effects in a more realistic shallow-water ocean environment. Both of the analytical and numerical calculations predict anomalous sound radiation fields at locations far from the termination of a truncated internal-wave duct. The radiation patterns are unique to each mode and show strong spatial and temporal variability. The studies also show that the cause of this is a strong dependence on the position of an acoustic source in the duct, which changes in time for a moving wave and a moving or fixed source. Broadband simulations made in this paper reveal situations where one or more modes are completely absent on a fixed VLA because the strongly localized modal radiation beams cannot just 
reach the fixed array and mode multipath arising from dispersive propagation within the horizontal waveguide.

\section{ACKNOWLEDGMENTS}

The 3D PE program used in this paper was derived from a trial version written by Dr. John Colosi of the Naval Postgraduate School, CA, USA, and the authors acknowledge his valuable efforts to the original code. The authors must also thank all of SW06 participants for their efforts to make this large multi-disciplinary shallow-water ocean experiment successful; heartfelt thanks must be offered to the acoustics and physical oceanography program managers from the Office of Naval Research (ONR), Ellen Livingston and Terry Paluszkiewicz, for their support. This work was supported under ONR Grant No. N00014-05-1-0482 and the ONR postdoctoral fellowship award, Grant No. N00014-08-1-0204.

${ }^{1}$ B. G. Katsnel'son and S. A. Pereselkov, "Low-frequency horizontal acoustic refraction caused by internal wave solitons in a shallow sea," Acoust. Phys. 46, 684-691 (2000).

${ }^{2} \mathrm{R}$. Burridge and H. Weinberg, "Horizontal rays and vertical modes," in Wave Propagation and Underwater Acoustics, edited by J. B. Keller and J. S. Papadakis (Springer, Berlin, 1977), pp. 86-152.

${ }^{3}$ R. Oba and S. Finette, "Acoustic propagation through anisotropic internal wave fields: Transmission loss, cross-range coherence, and horizontal refraction," J. Acoust. Soc. Am. 111, 769-784 (2002).

${ }^{4}$ D. Lee and M. H. Schultz, Numerical Ocean Acoustic Propagation in Three Dimensions (World Scientific, Singapore, 1995).

${ }^{5}$ S. Finette and R. Oba, "Horizontal array beamforming in an azimuthally anisotropic internal wave field," J. Acoust. Soc. Am. 114, 131-144 (2003).

${ }^{6}$ M. Badiey, B. G. Katsnelson, J. F. Lynch, S. Pereselkov, and W. L. Siegmann, "Measurement and modeling of three-dimensional sound intensity variations due to shallow-water internal waves," J. Acoust. Soc. Am. 117, 613-625 (2005).

${ }^{7}$ S. D. Franks, M. Badiey, J. F. Lynch, and W. L. Siegmann, "Experimental evidence of three-dimensional acoustic propagation caused by nonlinear internal waves," J. Acoust. Soc. Am. 118, 723-734 (2005).

${ }^{8}$ D. B. Reeder, T. F. Duda, and B. Ma, "Short-range acoustic propagation variability on a shelf area with strong nonlinear internal waves," in Oceans'08 (Kobe) Conference Proceedings (IEEE, New York, 2008).

${ }^{9}$ C. R. Jackson, An Atlas of Internal Solitary-Like Waves and Their Properties, 2nd ed. (Global Ocean Associates, Alexandria, VA, 2004) (available at http://www.internalwaveatlas.com/Atlas2_index.html (Last viewed 6/17/2009).
${ }^{10}$ T. F. Duda, "Initial results from a Cartesian three-dimensional parabolic equation acoustical propagation code," Technical Report No. WHOI-2006041, Woods Hole Oceanographic Institution, Woods Hole, MA, 2006.

${ }^{11}$ D. J. Tang, J. N. Moum, J. F. Lynch, P. Abbot, R. Chapman, P. H. Dahl, T. F. Duda, G. Gawarkiewicz, S. Glenn, J. A. Goff, H. Graber, J. Kemp, A. Maffei, J. D. Nash, and A. Newhall, "Shallow Water '06, A joint acoustic propagation/nonlinear internal wave physics experiment," Oceanogr. 20, 156-167 (2007).

${ }^{12}$ J. C. Preisig and T. F. Duda, "Coupled acoustic mode propagation through continental-shelf internal solitary waves," IEEE J. Ocean. Eng. 22, 256269 (1997).

${ }^{13}$ J. Zhou, X. Zhang, and P. H. Rogers, "Resonant interaction of sound wave with internal solitons in the coastal zone," J. Acoust. Soc. Am. 90, 20422054 (1991).

${ }^{14} \mathrm{H}$. Levine and J. Schwinger, "On the radiation of sound from an unflanged circular pipe," J. Acoust. Soc. Am. 73, 383-405 (1948).

${ }^{15}$ F. B. Jensen, W. A. Kuperman, M. B. Porter, and H. Schmidt, Computational Ocean Acoustics (AIP, Woodbury, NY, 1994).

${ }^{16} \mathrm{G}$. V. Frisk, Ocean and Seabed Acoustic: A Theory of Wave Propagation (Prentice-Hall, Englewood Cliffs, NJ, 1994).

${ }^{17}$ R. H. Hardin and F. D. Tappert, "Applications of the split-step Fourier method to the numerical solution of nonlinear and variable coefficient wave equations," SIAM Rev. 15, 423 (1973).

${ }^{18}$ D. J. Thomson and N. R. Chapman, "A wide-angle split-step algorithm for the parabolic equation," J. Acoust. Soc. Am. 74, 1848-1854 (1983).

${ }^{19}$ T. F. Duda, "Analysis of finite-duration wide-band frequency sweep signals for ocean tomography," IEEE J. Ocean. Eng. 18, 87-94 (1993).

${ }^{20}$ M. B. Porter, "The KRAKEN normal mode program," SACLANTECN Memorandum No. SM-245 (SACLANT Undersea Research Centre, La Spezia, Italy, 1991).

${ }^{21}$ Y.-M. Jiang, N. R. Chapman, and M. Badiey, "Quantifying the uncertainty of geoacoustic parameter estimates for the New Jersey self by inverting air gun data," J. Acoust. Soc. Am. 121, 1879-1894 (2007).

${ }^{22}$ T. F. Duda, "Examining the validity of approximations to fully threedimensional shallow-water acoustic propagation through nonlinear gravity waves," in Oceans'07 (Aberdeen) Conference Proceedings (IEEE, New York, 2007).

${ }^{23}$ W. M. Carey, J. Doutt, R. B. Evans, and L. M. Dillman, "Shallow-water sound transmission measurements on the New Jersey continental shelf," IEEE J. Ocean. Eng. 20, 321-336 (1995)

${ }^{24}$ R. J. Cederberg, W. M. Carey, and W. L. Siegmann, "Modal analysis of geoacoustic influences on shallow-water propagation," IEEE J. Ocean. Eng. 22, 237-244 (1997).

${ }^{25}$ J. M. Collis, T. F. Duda, J. F. Lynch, and H. A. Deferrari, "Observed limiting cases of horizontal field coherence and array performance in a time-varying internal wave field," J. Acoust. Soc. Am. 124, EL97-EL103 (2008). 